\title{
General factors governing the efficiency of luminescent devices
}

\author{
(C) William M. Yen \\ Department of Physics and Astronomy, University of Georgia, \\ Athens, GA 30602, USA \\ E-mail: wyen@hal.physast.uga.edu, \\ wyen@physics.wustl.edu
}

\begin{abstract}
We discuss in general terms, factors which determine the luminescence efficiency of activated wide band gap materials. These materials find their usage in a large variety of luminescent devices. The factors affecting the efficiency can be classified into two broad categories: the first has been thoroughly studied and entails interactions which lead to non-radiative dissipation of optical energy at the specific active site. The second set concerns interactions leading to the delocalization and the transport of optical energy away from the originally excited state. Recent spectroscopic results from these studies have allowed the absolute placement of the ground state of the active centers with respect to the intrinsic bands of the host crystal and have allowed us to elucidate the systematics of such placement. These results are relevant to materials which evince the property of persistent phosphorescence and which are of our current interest. Developments in these materials as well as a number of new applications will be touched upon.
\end{abstract}

This work was supported by the National Science Foundation and by the University of Georgia Research Foundation.

\section{Introduction}

In this article, we discuss factors which affect the luminescence efficiency of impurity activated insulators; though Professor Feofilov did not address this specific topic directly, his contributions to our understanding of the spectroscopy of impurity and defect activated system necessarily provided the basis for our understanding of this subject. This article, then, is prepared as a tribute to his memory and to his influence on all of us engaged in this area of scientific inquiry. Much progress has already been made in delineating the physical processes governing luminescent efficiency of light emitting materials; we now believe that the interactions affecting efficiencies may be classified into two broad categories. The first set is localized and is specific to the active center or ion, the second category arises from inter-ionic interactions and results in delocalization, transport and/or trapping of energy. By reclassifying in this manner, we have gained new insights with which to address the efficiency problem; as a bonus, we have developed the means with which to determine the absolute positioning of the impurity energy levels of the active centers within the host band gap. We will discuss some recent applications in which we can exploit this better understanding.

\section{Luminescence efficiencies}

The static spectra of impurity activated insulators can be understood in terms of the Coulomb interactions (crystalline field) produced by the neighboring host ions which lead to the shifting and splitting of the ion wave functions and determine the nature of the radiative transitions within the active ion. For the most common activators, i.e. ions belonging to the $(3 d)^{n}$ and $(4 f)^{n}$ ions series, the majority of luminescent transitions arise between states belonging to the same atomic configuration and hence are LaPorte or parity forbidden. Nevertheless, radiative transitions occur because the crystalline field can admix high lying allowed states into the interacting levels; the magnitude of this admixture is responsible to a large extent for the strength of the optical transition. Thus, because of the energy denominators, the energy placement of the atomically allowed states is one of the principal factors in determining the intrinsic light emission efficiency of a material and the determination of the energy of these states constitutes a predictor of efficiencies [1].

Further, the ion and the host lattice system are dynamically coupled and the intrinsic excitations of the lattice, such as phonons, can induce non-radiative relaxation processes in which optical excitation is dissipated through the creation of vibrational or other intrinsic excitations. These relaxation pathways, of course, have a deleterious effect on the radiative efficiencies; it is these processes, for example, which severely limit the number of metastable, emissive states in the systems of interest here. The radiative and nonradiative properties of impurity ions in solids has been the subject of intensive scrutiny in the past few decades and we need not revisit the subject in detail here [2].

In our new reclassified picture, we consider these intraionic and ion-phonon interactions to be of a localized nature inasmuch as they are confined to the individual excited center.

\section{Delocalization of energy}

Recent work has lead us to conclude that a second class of processes must also be consider and that this class plays an important and sometimes crucial role in determining the luminescence efficiency. These processes are ulterior to the individual centers and lead to the delocalization of optical energy through transfer or through transport. Inter-ionic multipolar interactions, for example, lead to ion-ion transfer processes which can cause cross relaxation and diffusion of the optical energy [2]. 


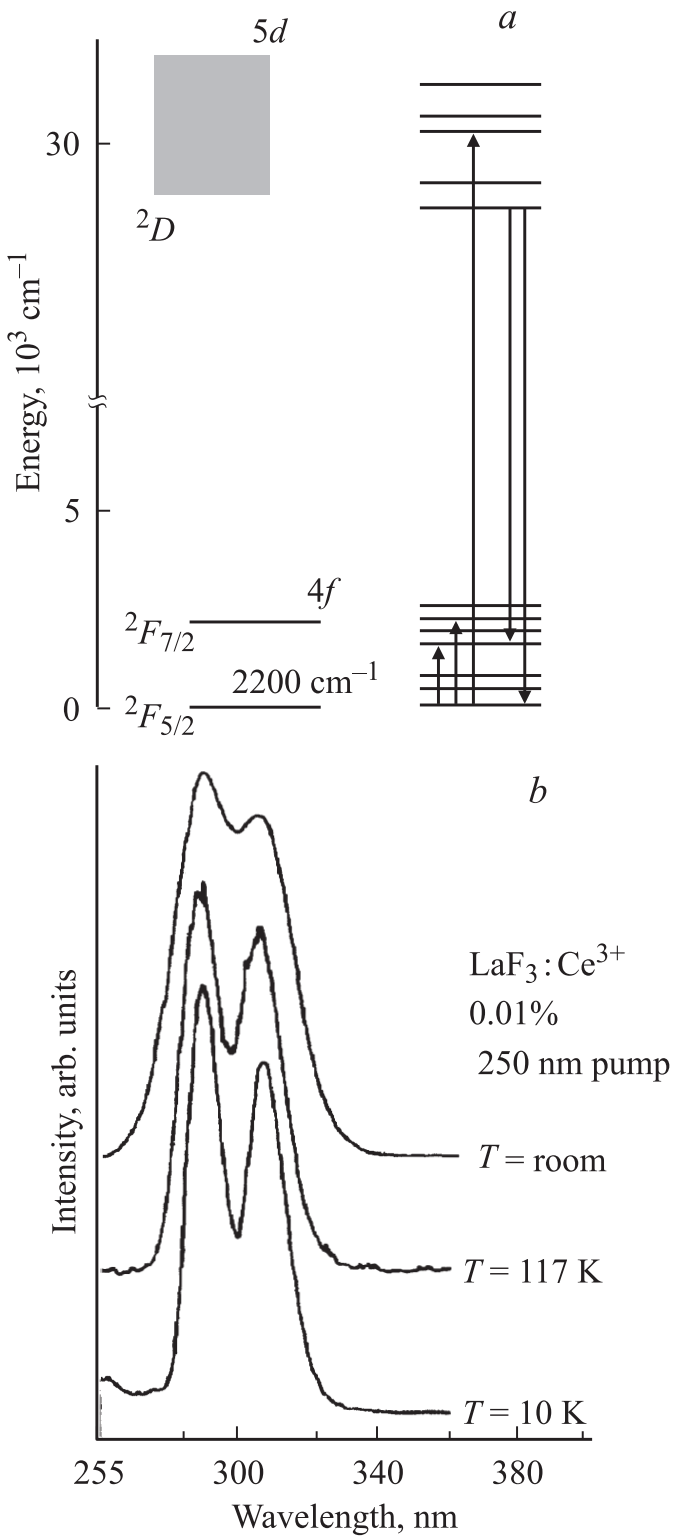

Figure 1. Schematic diagram of the energy levels of $\mathrm{Ce}^{3+}(a)$. The splitting of the ground state is approximately $2500 \mathrm{~cm}^{-1}$; the $5 d$ states are affected by the crystalline field and emission is observed from the lowest $d$ component. The first observed Ce luminescence was in $\mathrm{LaF}_{3}$ obtained through excitation using synchrotron radiation; the luminescence as a function of temperature is shown in part $(b)[3]$.

The importance of the second class of processes will become evident when we consider the luminescence behavior of a simple system, i.e. $\mathrm{Ce}^{3+}$ activated insulators. $\mathrm{Ce}^{3+}$ belongs to the $(4 f)^{1}$ configuration and possesses a very simple energy level structure shown in Fig. 1, $a$ consisting of a ground state doublet $\left({ }^{2} F_{5,7 / 2}\right)$ separated by a few thousand wave-numbers and an allowed $5 d$ state lying in the near $U V$ region of the spectra. The $4 f$ to $5 d$ transition is allowed; a strong absorption band is observed which reflects the crystalline field induced effects on the $5 d$ state. A strong luminescence originating from the lowest $\mathrm{Ce}^{3+} 5 d$ component was first observed in $\mathrm{LaF}_{3}$ and is also shown in Fig. 1, $b$ [3]. When excited, the energy structure of $\mathrm{Ce}$ is such that localized multiphonon non-radiative relaxation and transfer and cross relaxation quenching to other like ions are highly improbable; the expectation then is be that invariably $5 d$ Ce luminescences will be strong and highly efficient.

Emission of $\mathrm{Ce}^{3+}$ doped compounds

\begin{tabular}{c|c}
\hline Efficient & Quenched \\
\hline $\mathrm{Y}_{3} \mathrm{Al}_{5} \mathrm{O}_{12}(\mathrm{YAG})$ & Nitrates \\
$\mathrm{LuAlO}_{3}(\mathrm{LuAP})$ & Vanadates \\
$\mathrm{YAlO}_{3}(\mathrm{YALO})$ & Tungstates \\
$\mathrm{Lu}_{2}, \mathrm{Y}_{2}\left(\mathrm{SiO}_{4}\right) \mathrm{O}$ & Lanthanum oxide \\
$\mathrm{CaF}_{2}, \mathrm{LaF}_{3}$ & Gadolinium oxide
\end{tabular}

In a study of the properties of $\mathrm{Ce}^{3+}$ in a variety of host lattices, it came as a surprise to us that though strong emissions were observed in some lattices, no $5 d$ luminescences were observed in some others (see the Table). For our purposes, it is interesting to compare and contrast the behavior of $\mathrm{Ce}^{3+}$ in two lattices from the Table, $\mathrm{LuSiO}_{5}(\mathrm{LSO})$ and $\mathrm{Lu}_{2} \mathrm{O}_{3}$, where the band gaps of the hosts are about the same. In both cases, $\mathrm{Ce}^{3+}$ replaces $\mathrm{Lu}^{3+}$ and their local coordination is also roughly analogous. As the Table shows, $\mathrm{Ce}^{3+}$ in LSO luminesces strongly whereas no $5 d$ emission is observed in $\mathrm{Lu}_{2} \mathrm{O}_{3}: \mathrm{Ce}^{3+}[4]$.

The optical spectra of these two materials are shown in Figs. 2 and 3. The structure of the absorption and/or excitation spectra in both cases are identifiable as field components of the respective $5 d$ states; the Stokes shifted luminescence of $\mathrm{LSO}: \mathrm{Ce}^{3+}$ originates from the lowest $d$ state of the manifold and reflects the ground state structure of the cerium ion, also as expected. Since there is no emission at any temperature, Fig, 3 shows the absorption of pure as well as trivalent and tetravalent $\mathrm{Ce}$ doped $\mathrm{Lu}_{2} \mathrm{O}_{3}$.

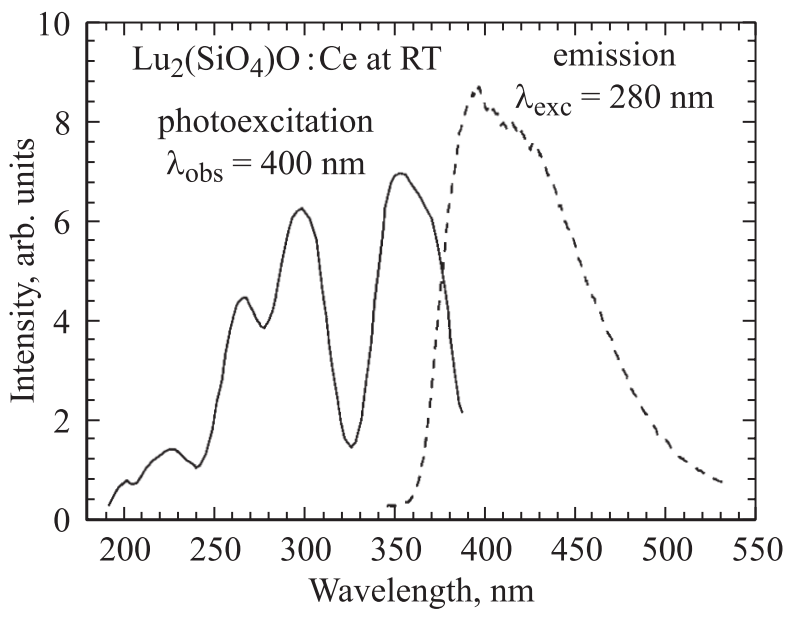

Figure 2. $\mathrm{Ce}^{3+}$ luminescence observed in $\mathrm{Lu}_{2} \mathrm{SiO}_{5}$ (LSO); the excitation spectrum for the emission is also shown and reflects the crystalline field effects on the $5 d$ manifold. 


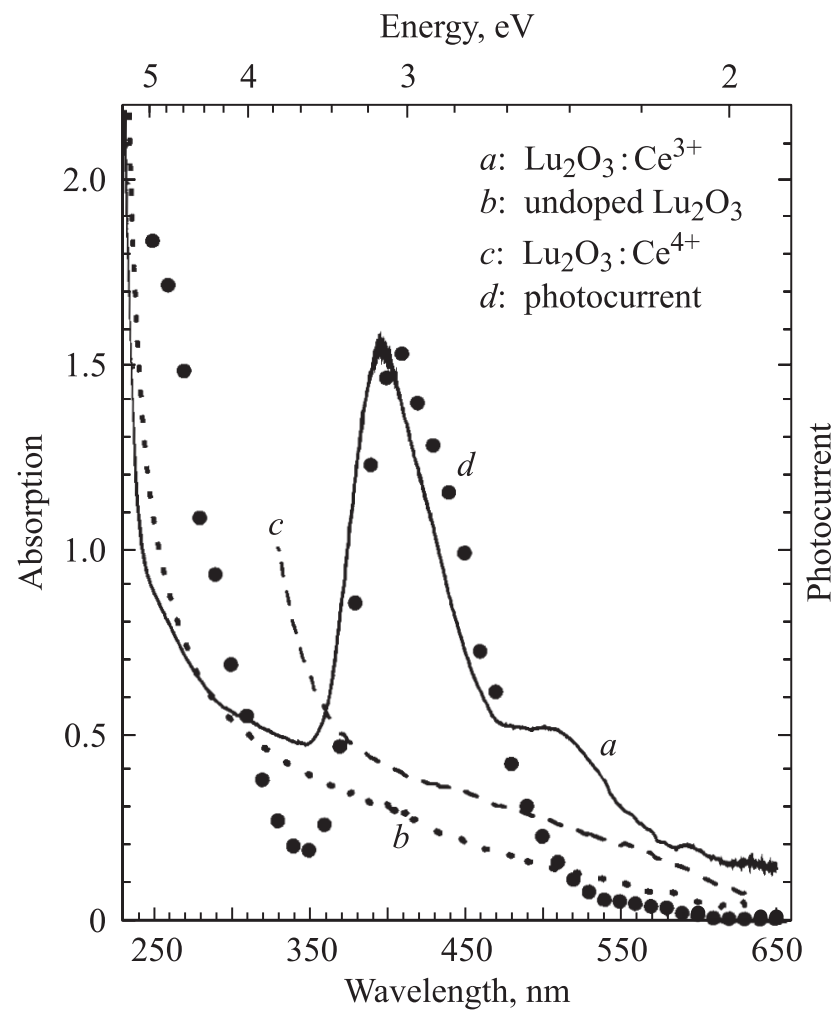

Figure 3. The absorption and the $\mathrm{PC}$ spectra of $\mathrm{Lu}_{2} \mathrm{O}_{3}: \mathrm{Ce}^{3+}$. The absorption spectra of pure $\mathrm{Lu}_{2} \mathrm{O}_{3}$ and $\mathrm{Lu}_{2} \mathrm{O}_{3}: \mathrm{Ce}^{4+}$ are also shown. This system does not show any $5 d$ luminescence. It is seen here that the PC spectrum closely follows the $5 d$ absorption in the trivalent $\mathrm{Ce}$ compound. The energy shift observed in the PC spectrum can be ascribed to time constant effects.

Photoconductivity (PC) measurements were conducted on these two compounds which revealed the reason for their drastically different emission efficiencies. In PC spectroscopy one measures the creation of mobile electronic charges into the conduction band from the valence band or from the photoionization of impurities. The measured PC spectrum for $\mathrm{Lu}_{2} \mathrm{O}_{3}: \mathrm{Ce}^{3+}$ is shown in Fig. 3 plotted against the $5 d$ absorption; it can be seen from there that the PC spectra track the absorption faithfully; this implies that promotion of $\mathrm{Ce}$ electrons from the ground state to the $5 d$ state is akin to promoting charges directly into the conduction band. It follows that in this case all the field components of the $5 d$ configuration fall within the conduction band of the host and electrons promoted to these energies become undistinguishable from free conduction electrons. The fact that electrons in the conduction band are mobile then implies that the optical energy is no longer localized at a single ion site [5].

Fig. 4 shows the PC observed in LSO: $\mathrm{Ce}^{3+}$ showing that the $\mathrm{PC}$ signal is much smaller and that it is temperature activated. The case for LSO can be understood in terms of the schematic shown of the inset. All $5 d$ states except the lowest level lie within the conduction band and optical energy can be stored in this lowest $5 d$ level and radiated; the observed PC and its temperature dependence results simply from thermal activation from the radiative state to the conduction band [6]. Basically, the difference between the performance of these two materials lies in the placement of the $5 d$ states relative to conduction band; the absolute
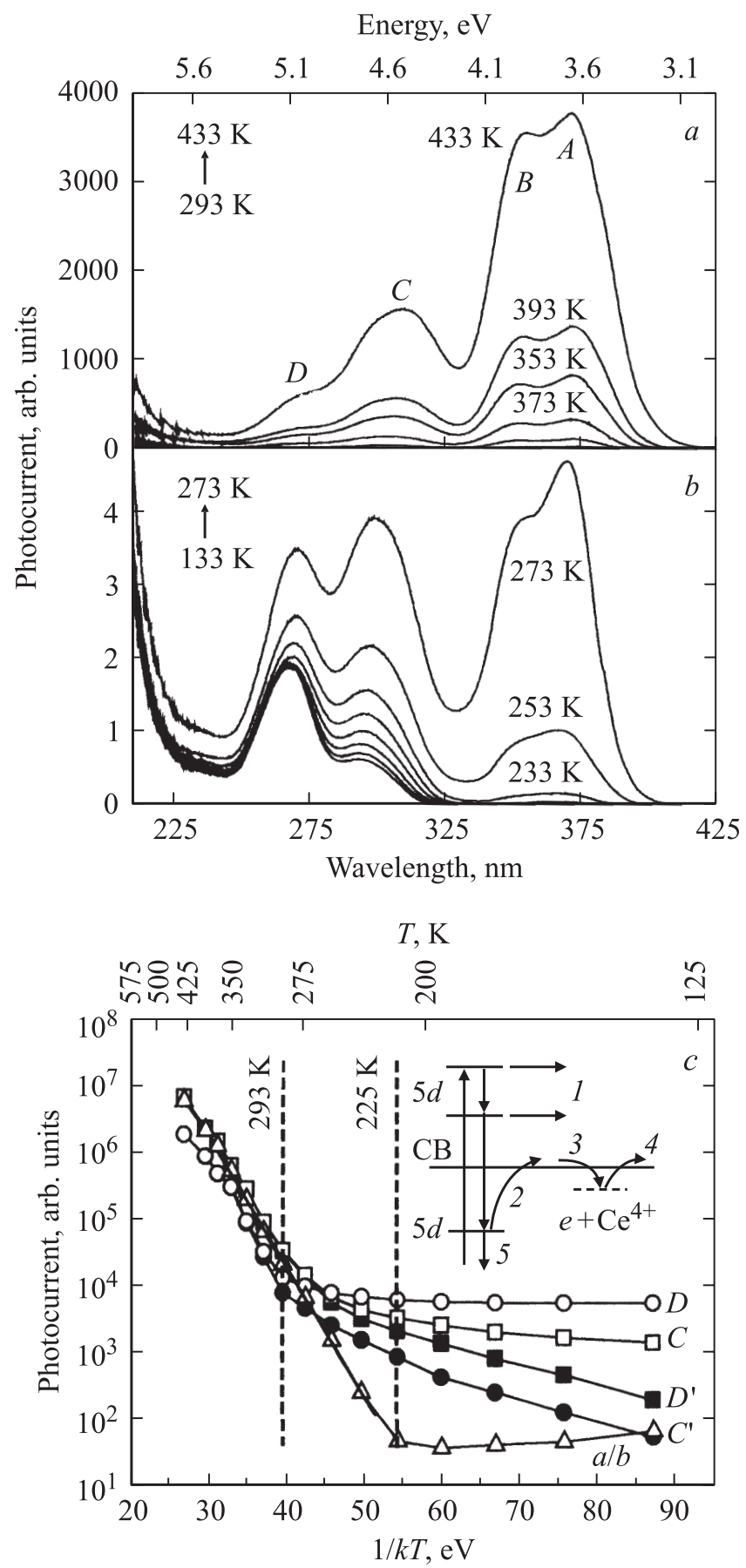

Figure 4. The $\mathrm{PC}$ spectra observed from $\mathrm{Ce}$ in two different sites of LSO $(a, b)$. The PC peaks labeled $A, B, C, \ldots$ represent the structure of the $5 d$ band of the $\mathrm{Ce}$ in their respective sites. The PC plotted against temperature $(c)$ clearly indicate a thermally activated behaviour. These plots for various PC peaks allow us to determine the placement of the $5 d$ components relative to the conduction band edge [6]. 
placement of the energy levels of the active center viz the intrinsic bands and the band gap of the host then can be seen to play a critical role in determining the emission efficiencies through the process of delocalization of the optical energy.

\section{Localization/delocalization}

In order to show that our interpretation of the contrast in behavior is correct, we have investigated the behavior of $\mathrm{Lu}_{2} \mathrm{O}_{3}: \mathrm{Ce}^{3+}$ as a function of hydrostatic pressure. The rationale behind these experiments is shown in Fig. 5; hydrostatic pressure of the magnitudes attainable with a diamond anvil cell decrease the dimensions of the unit cell of the crystal and as a consequence increase the crystalline field strength. The splitting of the $5 d$ manifold is then expected to increase with the lowest $5 d$ component moving to lower energies while keeping the center of gravity of the $5 d$ manifold unchanged. If the pressure is sufficiently high, a crossover of this $5 d$ state with the conduction band edge will occur. Under these circumstances, the energy level scheme of $\mathrm{Lu}_{2} \mathrm{O}_{3}: \mathrm{Ce}^{3+}$ begins replicating that encountered in LSO, i.e. luminescence from the lowest $5 d$ state will occur. Also in analogy to LSO, the pressure induced lowest $5 d$ state in the oxide will be subject to quenching through thermal activation back into the conduction band [7].

Recalling that $\mathrm{Lu}_{2} \mathrm{O}_{3}: \mathrm{Ce}^{3+}$ shows no luminescence at ambient pressure, a result from of pressure studies is shown

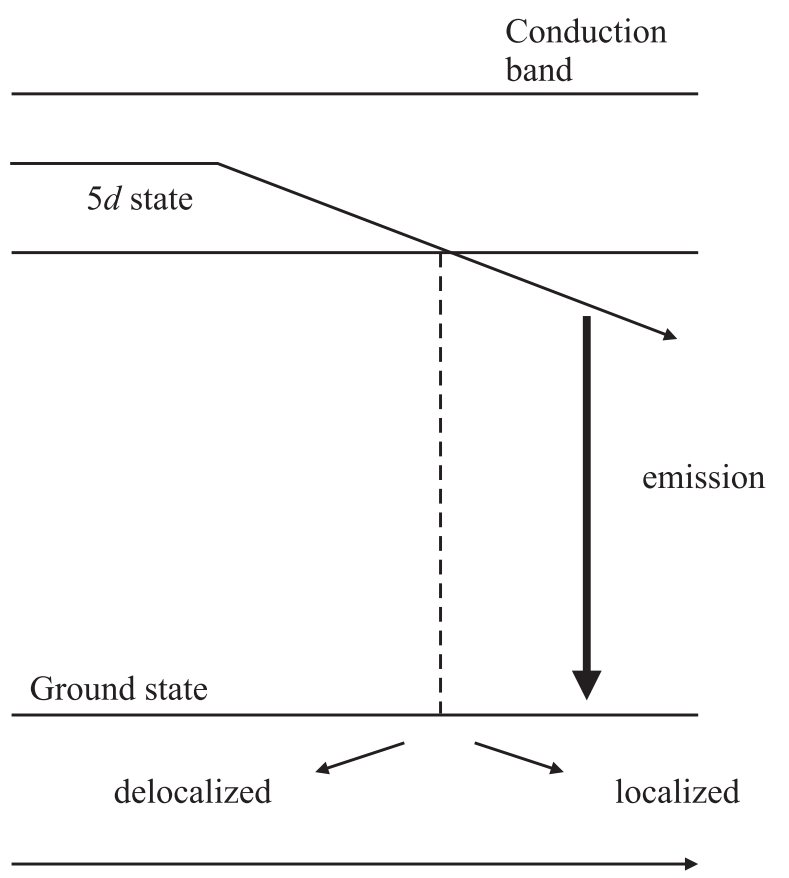

Pressure increase, kbar

Figure 5. Rationale behind the high pressure experiments on $\mathrm{Lu}_{2} \mathrm{O}_{3}: \mathrm{Ce}^{3+}$ showing the crossover of the lowest $5 d$ state and the bottom of the conduction band. This crossover results in the emergence of $5 d$ luminescence and signals the localization of the optical energy.
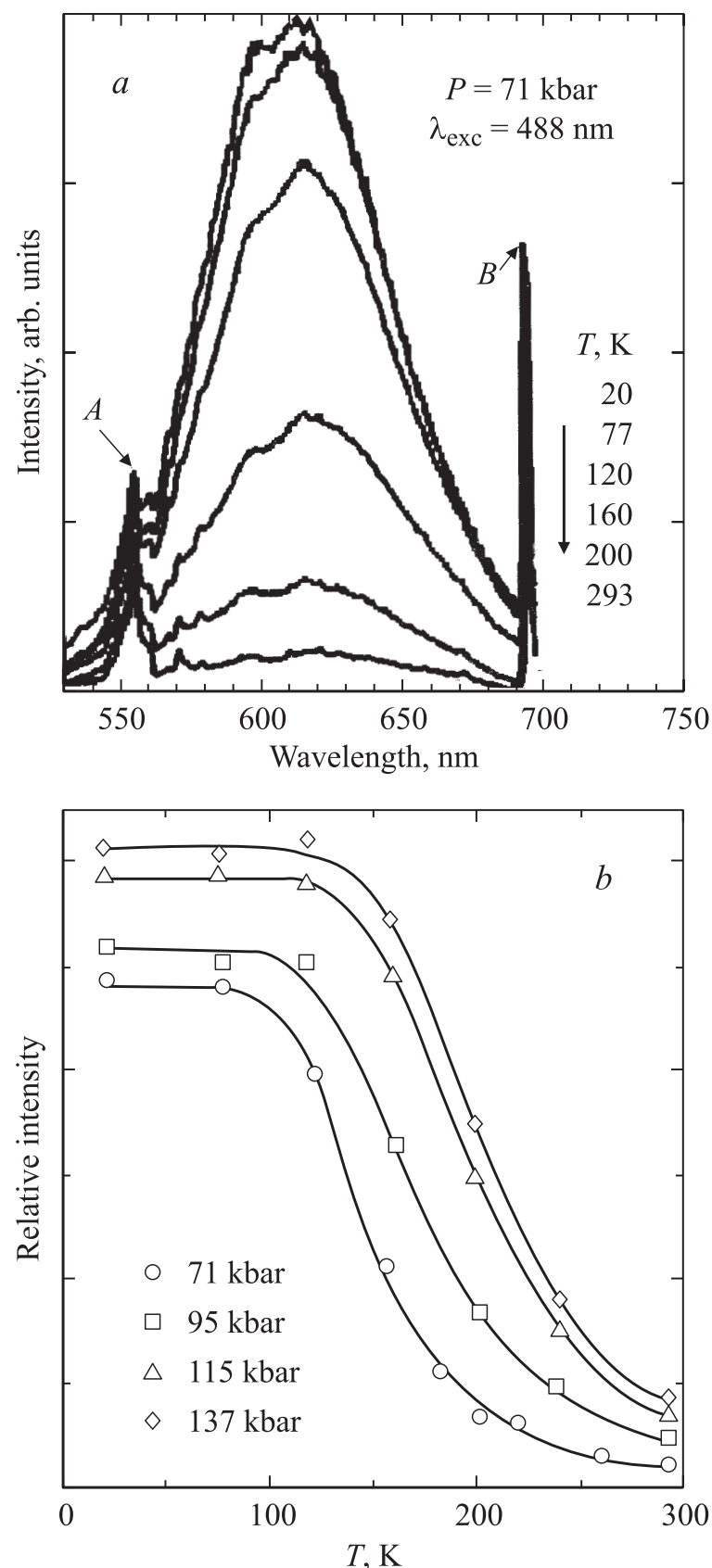

Figure 6. $a$ - temperature dependence of the pressure induced luminescence in $\mathrm{Lu}_{2} \mathrm{O}_{3}: \mathrm{Ce}^{3+}$. Pressure is $71 \mathrm{kbars}$. The sharp lines shown in figure arise from the anvil cell and from a ruby monitor. $b$ - integrated emission intensities as a function of pressure. The transition edge allows the positioning of the $5 d$ state as a function of pressure [7].

in Fig. 6. Fig. 6, $a$ shows the luminescence observed in the oxide material when a pressure of $71 \mathrm{kbar}$ is applied at various temperatures. The behavior of the luminescence is seen to be similar to that observed in LSO and with thermal quenching also apparent. Thermal activation curves such as those shown in Fig. 6, $b$, allow the determination of the position of the $5 d$ level below the band edge. 
As argued in an earlier section, the luminescence of the $5 d$ state of $\mathrm{Ce}^{3+}$ signals the localization of the optical energy on a specific ion site whereas the overlap of the $5 d$ states with the conduction band results in the delocalization and transport of the energy through conduction band. It follows that our experiments, in which we induce a level crossing between a $5 d$ state and the conduction band edge, correspond to a transition between localized and delocalized states of the electronic excitations. This type of order disorder transitions have been considered previously in some detail and is related to the so-called Anderson transition; in other words, what our pressure experiments illustrate is an Anderson transition in the optical energy as affected by the transport properties of electronic carriers [8]. We would expect that the appearance of luminescence as a function of pressure will evince a sharp edge (the mobility edge); though our pressure control has not been sufficiently refined to allow the determination of such an edge, its observation is certainly a worthwhile goal to pursue.

\section{Placement of energy levels}

It should be apparent that the placement of the high lying energy levels of luminescent centers relative to the conduction band of the host plays an important role in determining the luminescence efficiencies of activated systems; hence it is important to be able to locate the ground state of the active ions absolutely within the band gap of the host material. The placement of the levels may be accomplished by considering factors such as those represented in Fig. 3 for $\mathrm{Lu}_{2} \mathrm{O}_{3}$; the absorption of the undoped lattice, of course, corresponds to the band gap of the material. The tetravalent $\mathrm{Ce}$ ion has a closed shell structure similar to $\mathrm{Xe}$ and hence is expected to have absorptive transitions far into the $U V$. The absorption beginning at $350 \mathrm{~nm}$ for the $\mathrm{Ce}^{4+}$ material, thus, arises because of an acceptor-like charge transfer process from the valence band of the host to the tetravalent ion converting it into a trivalent ion in the ground state. This fact coupled to the absorption due to the $5 d$ states of $\mathrm{Ce}^{3+}$ allows us to make an absolute energy placement for the ground state [9]. Such a placement for the ground of $\mathrm{Ce}^{3+}$ is illustrated in Fig. 7, $a$ for $\mathrm{Y}_{2} \mathrm{O}_{3}: \mathrm{Ce}^{3+}$.

It has become clear that the absolute placement of the ground state of impurity ions in solids relative to the intrinsic bands of the host has been an often neglected but essential component in our understanding of the total behavior of emissive properties of impurity ion systems. These problems have only begun to be addressed recently and several experimental techniques have been developed for these determination. $\mathrm{PC}$ has been shown to be useful in establishing the position of states from which conductivity may be induced; often, however, intrinsic bands and charge transfer processes complicate the interpretation of these results. In addition to PC studies, photoelectron spectroscopy has also been used to determine the position
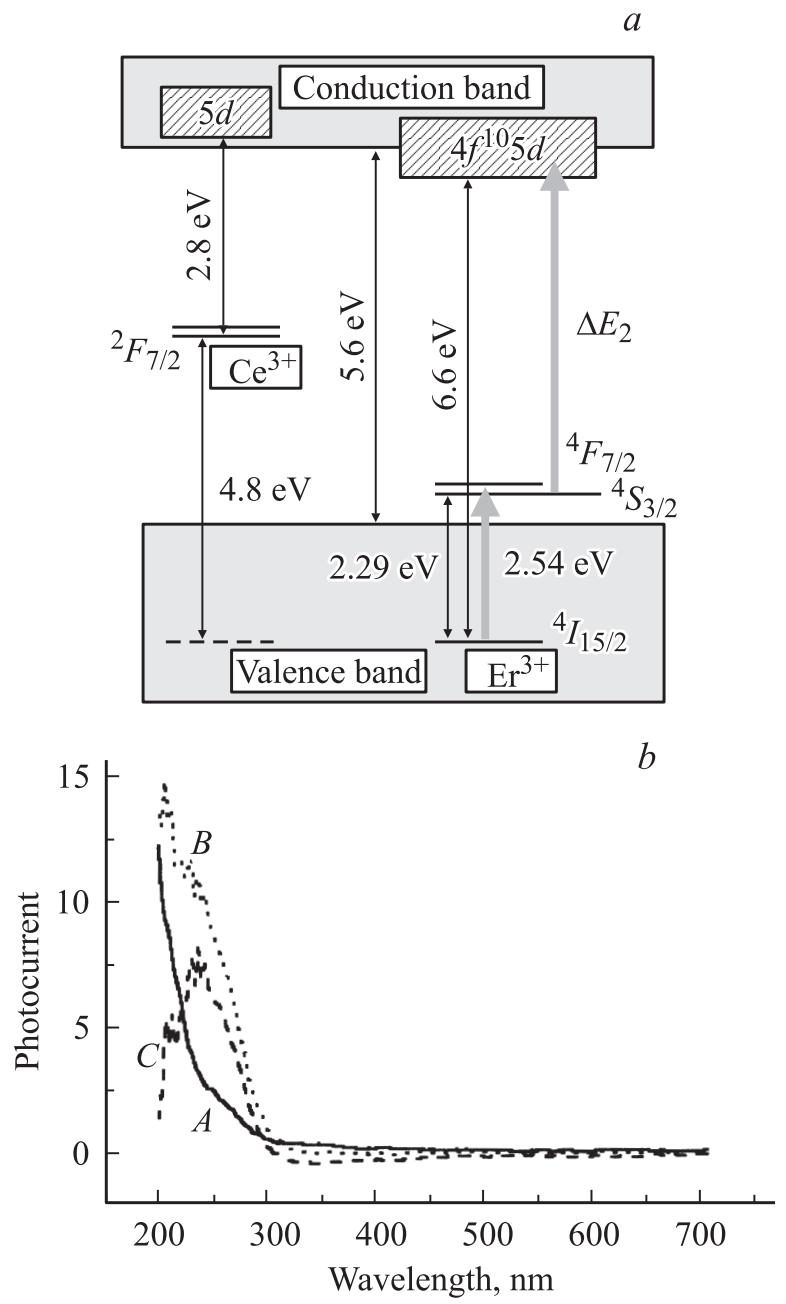

Figure 7. Placement of the ground state of $\mathrm{Ce}^{3+}$ and $\mathrm{Er}^{3+}$ in $\mathrm{Y}_{2} \mathrm{O}_{3}$ using two excitation photons and inducing photoconductivity from a metastable state $(a)$ [11]. PC spectra of $\mathrm{Er}^{3+}$ in $\mathrm{Y}_{2} \mathrm{O}_{3}(b)$. $A$ - PC observed with $U V$ lamp only showing ground state and intrinsically induced currents; $B-\mathrm{PC}$ observed with lamp and laser excitation showing currents induced from the ground and metastable states; $C$ - difference spectra showing contributions from the ${ }^{4} S_{3 / 2}$ state only allowing the placement of the ground state as in part $(a)$.

of the ground state of the $4 f$ ion series in a number of lattices [10]; these studies have shown that in some of the rare earth ion ground states fall within the valence band of the host.

We have recently developed a new PC based technique which reduces the contributions from sources other than the center of interest. These two step experiments are illustrated in Fig. 7, $a$ and entail the measurement of photoconductivity from selectively designated excited states of specific impurity centers, i.e. excited state PC [11]. The Figure illustrates two-step PC measurements in $\mathrm{Y}_{2} \mathrm{O}_{3}: \mathrm{Ce}^{3+}$ (as noted above) and $\mathrm{Y}_{2} \mathrm{O}_{3}: \mathrm{Er}^{3+}$; in the latter, the first laser photon selectively excites the $\mathrm{Er}$ ion from its ${ }^{4} I_{15 / 2}$ ground state to the metastable ${ }^{4} S_{3 / 2}$ state. A second photon then is 
used to excite the ion to its $5 d$ states or into the conduction band of the host to induce the photocurrent; comparison of the PC spectra under direct and two step excitation provides sufficient information to allow the absolute placement of the ground state of the $\mathrm{Er}^{3+}$ ion relative to the intrinsic bands of the host as is indicated in Fig, 7, $b$. As can be seen from the figure, the $\mathrm{Er}^{3+}$ ground state actually falls within the valence band of the host $\mathrm{Y}_{2} \mathrm{O}_{3}$.

Recently, Dorenbos has conducted an exhaustive survey of the systematics of the placement of the energy levels of the $4 f$ ion series in large band gap solids [12]. He exploits trends observed in the ionization behavior of the ions in the series and focuses on the shared properties of certain common transitions of the ions in order to deduce the positioning of the ground states. He assumes, for example, that the energy necessary to ionize ions into a higher valence through charge transfer is roughly the same across the $4 f$ ion series. Using these arguments, he has been successful in establishing the ground state position of the $4 f$ ion series in various insulating materials. His results generally agree with the experimental observations and signal that we are reaching a qualitative understanding of one of the last remaining problem in the spectral behavior of impurity activated insulators.

\section{Applications and conclusions}

As we gain a more detailed knowledge of the absolute placement of impurity ions within large band gap materials, we can better predict the behavior of these materials from different points of view; this includes the consideration of the overall performance efficiency of luminescent materials. For example, there has been considerable recent interest in phosphors which display phosphorescence persisting for tens of hours. One such material is $\mathrm{SrAl}_{2} \mathrm{O}_{4}: \mathrm{Eu}^{2+}$, $\mathrm{Dy}^{3+}$ where a strong green emission arising from the $d$ to $f$ transition of $\mathrm{Eu}^{2+}$ is observed persisting for nearly twenty hours following excitation. The persistence has been ascribed to a mechanism in which the $\mathrm{Dy}^{3+}$ ions trap holes, creating excess electrons which can then transfer their energy to an $\mathrm{Eu}^{2+}$ emitter. The trapping behavior of various ions in the $4 f$ series may be deduced from the energy trends derived from the energy systematics; the proximity of the $\mathrm{Dy}^{3+}$ ground state to the valence band, for example, favors hole trapping. Substitution of $\mathrm{Tb}^{3+}$ for $\mathrm{Dy}^{3+}$ in this aluminate destroys the persistence, $\mathrm{Tb}^{3+}$ is more likely to trap electrons [13]. We believe that the above developments will likely influence the way we look at electroluminescence behavior.

As a summary, we have classified two types of processes which affect the overall luminescent efficiency of luminescent materials such as phosphors. Some factors which have been ignored in these considerations include interactions which lead to the delocalization of optical energy away from the active center or ion and lead to the diffusion and trapping of energy. The physical boundary between the maintenance of energy at a site and its delocalization constitutes an Anderson transition which should display a mobility edge. Recently, we have also begun to understand the systematics which govern the placement of the impurity energy levels within the band gap of the host and allowed us to better predict the behavior and efficiency of phosphors.

The contributions to this work from present and past member of my Georgia group are acknowledged with gratitude. The hospitality of Washington University at St. Louis during the preparation of this manuscript is acknowledged with thanks.

\section{References}

[1] G. Blasse, B.C. Grabmaier. Luminescent Materials. Springer, Berlin (1994).

[2] B. Henderson, G.F. Imbusch. Optical Spectroscopy of Inorganic Solids. Science Publications, Oxford (1989).

[3] L.R. Elias, W.S. Heaps, W.M. Yen. Phys. Rev. B 8, 4989 (1973).

[4] Madis Raukas. Ph.D. Thesis. University of Georgia, 1997 (unpublished).

[5] W.M. Yen et al. J. Lumin. 69, 287 (1996).

[6] E. van der Kolk, S.A. Basun, G.F. Imbusch, W.M. Yen. Appl. Phys. Lett. 83, 1740 (2003).

[7] Yongron Shen et al. Phys. Rev. B 65, 212103 (2002).

[8] P.W. Anderson. Phys. Rev. 109, 1492 (1958).

[9] W.M. Yen. J. Lumin. 83/84, 399 (1999).

[10] C.W. Thiel et al. Phys. Rev. B 64, 085107 (2001).

[11] D. Jia, R.S. Meltzer, W.M. Yen. Phys. Rev. B 65, 235116 (2002).

[12] P. Dorenbos. J. Lumin. 108, 301 (2004).

[13] T. Matsuzawa et al. J. Electrochem. Soc. 143, 2670 (1996). 